\title{
The Fractional Fourier Transform and Its Application to Energy Localization Problems
}

\author{
Patrick J. Oonincx \\ Department of Nautical Sciences, Royal Netherlands Naval College (KIM), P.O. Box 10000, 1780 CA Den Helder, The Netherlands \\ Email:p.j.oonincx@kim.nl
}

Hennie G. ter Morsche

Department of Mathematics and Computer Science, Eindhoven University of Technology, P.O. Box 513, 5600 MB Eindhoven, The Netherlands

Email:morscheh@win.tue.nl

Received 20 March 2002 and in revised form 4 April 2003

\begin{abstract}
Applying the fractional Fourier transform (FRFT) and the Wigner distribution on a signal in a cascade fashion is equivalent to a rotation of the time and frequency parameters of the Wigner distribution. We presented in ter Morsche and Oonincx, 2002, an integral representation formula that yields affine transformations on the spatial and frequency parameters of the $n$-dimensional Wigner distribution if it is applied on a signal with the Wigner distribution as for the FRFT. In this paper, we show how this representation formula can be used to solve certain energy localization problems in phase space. Examples of such problems are given by means of some classical results. Although the results on localization problems are classical, the application of generalized Fourier transform enlarges the class of problems that can be solved with traditional techniques.
\end{abstract}

Keywords and phrases: fractional Fourier transform, Wigner distribution, symplectic transformation, energy localization.

\section{INTRODUCTION}

In this paper, we generalize the concept of the fractional Fourier transform (FRFT) as introduced by Kober [1] and show its application for solving certain energy localization problems in phase space. In the sequential sections, we will deal with the FRFT; however, here we briefly recall the definition and some properties of the Wigner distribution. This time-frequency representation is the most commonly used tool to analyse the FRFT, see, for example, [2]. Relations between fractional operators and other time-frequency distributions were studied in a general fashion in [3]. As is probably well known, the Wigner distribution for a signal $f$ with finite energy, that is, $f \in L^{2}(\mathbb{R})$, is given by

$$
\mathscr{W} \mathscr{V}[f](x, \omega)=\frac{1}{2 \pi} \int_{\mathbb{R}} f\left(x+\frac{t}{2}\right) \overline{f\left(x-\frac{t}{2}\right)} e^{-i t \omega} d t .
$$

Throughout this paper, we use the multidimensional mixed Wigner distribution that reads

$$
\mathscr{W} \mathscr{V}[f, g](x, \omega)=(2 \pi)^{-n} \int_{\mathbb{R}^{n}} f\left(x+\frac{t}{2}\right) \overline{g\left(x-\frac{t}{2}\right)} e^{-i(t, \omega)} d t
$$

for all $n$-dimensional functions $f$ and $g$ with finite energy, that is, $f, g \in L^{2}\left(\mathbb{R}^{n}\right)$, and with $(\cdot, \cdot)$ representing the inner product in $\mathbb{R}^{n}$. In the case $g=f$, we will use the short notation of the Wigner distribution $\mathscr{W}^{\mathscr{V}}[f]$. Here we briefly recall some properties of the mixed Wigner distribution, which are used throughout this paper.

The Wigner distribution is invariant under the action of both translation $\mathscr{T}_{b}$ and frequency modulation $\mathcal{M}_{\omega_{0}}$, given by $\mathscr{T}_{b}[f](x)=f(x-b)$ and $\mathcal{M}_{\omega_{0}}[f](x)=e^{i \omega_{0} x} f(x)$, for $b, \omega_{0} \in \mathbb{R}^{n}$ and $f$ acting on $\mathbb{R}^{n}$. A straightforward calculation shows that

$$
\begin{aligned}
\mathscr{W} \mathscr{V}\left[\mathscr{T}_{b} f\right](x, \omega) & =\mathscr{W} \mathscr{V}[f](x-b, \omega), \\
\mathscr{W} \mathscr{V}\left[\mathcal{M}_{\omega_{0}} f\right](x, \omega) & =\mathscr{W} \mathscr{V}[f]\left(x, \omega-\omega_{0}\right) .
\end{aligned}
$$

This means that a translation over $\left(x_{0}, \omega_{0}\right)$ in the Wigner plane, the phase space related to the Wigner distribution, corresponds to the operator

$$
\mathcal{N}_{\left(x_{0}, \omega_{0}\right)}[f](x)=T_{x_{0}} M_{\omega_{0}}[f](x)=e^{i\left(\omega_{0}, x\right)} f\left(x-x_{0}\right) .
$$

In relation to the FRFT, the following property is of importance. A rotation over $\pi / 2$ in all dimensions of the Wigner plane is achieved by the action of the Fourier transform $\mathscr{F}_{n}$ 
on the signal $f \in L^{2}\left(\mathbb{R}^{n}\right)$, that is,

$$
\mathscr{W} \mathscr{V}[\mathscr{F} f](x, \omega)=\mathscr{W} \mathscr{V}[f](-\omega, x) .
$$

For a comprehensive list of other properties of the Wigner distribution, we refer to $[4,5]$. One last property we want to mention here is the property of satisfying the time and frequency marginals, that is,

$$
\begin{aligned}
& |f(x)|^{2}=\int_{\mathbb{R}^{n}} \mathscr{W} \mathscr{V}[f](x, \omega) d \omega, \\
& |\hat{f}(\omega)|^{2}=\int_{\mathbb{R}^{n}} \mathcal{W V}^{\sigma}[f](x, \omega) d x .
\end{aligned}
$$

The sequel of this paper focuses on energy conserving (unitary) operators that correspond to classes of affine transformations in the Wigner plane. In Section 2, the FRFT is discussed as an operator that corresponds to rotation action in the Wigner plane. In Section 3, the whole class of affine transformations in the $n$-dimensional Wigner plane is presented and studied extensively. Also an integral representation for this class is presented. In Section 4, this representation is used in a mathematical framework for analyzing and solving energy localization problems in the Wigner plane. This framework is based on the Weyl correspondence. Finally, some examples of energy localization problems are discussed in Section 5. The framework of the latter section is used for solving two well-known energy localization problems.

\section{FRACTIONAL FOURIER TRANSFORM}

The FRFT on $L^{2}(\mathbb{R})$ was originally described by Kober [1] and was later introduced for signal processing by Namias [6] as a Fourier transform $(\mathscr{F})$ of fractional order, that is,

$$
\mathscr{F}_{\alpha} f=\mathscr{F}^{2 \alpha / \pi} f, \quad \forall_{f \in L^{2}(\mathbb{R})},
$$

for $\alpha \in[-\pi, \pi]$. From this formal definition, an integral representation for $\mathscr{F}_{\alpha}$ has been derived in a heuristic manner. Later this representation has been formalized in $[7,8]$. The integral representation for functions $f \in L^{2}(\mathbb{R})$ reads

$$
\mathscr{F}_{\alpha}[f](x)=\frac{C_{\alpha}}{\sqrt{2 \pi|\sin \alpha|}} \int_{\mathbb{R}} f(u) e^{i\left(\left(u^{2}+x^{2}\right) \cdot(\cot \alpha) / 2-u x \csc \alpha\right)} d u,
$$

for $0<|\alpha|<\pi$, with $C_{\alpha}=e^{i((\pi / 4) \operatorname{sgn} \alpha-\alpha / 2)}$. For $\alpha=0$ and $\alpha=\pi$, an expression for the FRFT follows directly from (8), namely, $\mathscr{F}_{0}[f](x)=f(x)$ and $\mathscr{F}_{\pi}[f](x)=f(-x)$. For $\alpha \notin$ $(-\pi, \pi]$, the FRFT is defined by periodicity $\mathscr{F}_{\alpha+2 \pi}=\mathscr{F}_{\alpha}$.

For time-frequency analysis, it is of interest to consider the relation of the FRFT with time-frequency operators like the Wigner distribution. In [2], Almeida showed that the FRFT $\mathscr{F}_{\alpha}$ gives raise to a rotation in the Wigner plane by an angle $\alpha$, that is,

$$
\mathscr{W} \mathscr{V}\left[\mathscr{F}_{\alpha} f\right](x, \omega)=\mathscr{W} \mathscr{V}[f]\left(R_{\alpha}(x, \omega)\right),
$$

where $R_{\alpha}(x, \omega)$ represents the matrix vector product with matrix

$$
R_{\alpha}=\left(\begin{array}{cc}
\cos \alpha & -\sin \alpha \\
\sin \alpha & \cos \alpha
\end{array}\right) .
$$

In particular, we have a rotation by $\pi / 2$ in the Wigner plane for $\mathscr{F}_{\pi / 2}$, which is a result that coincides with (5).

The action of the FRFT in the Wigner plane leads us in a natural way to the question, which operators on $L^{2}(\mathbb{R})$ act like a linear transformation in the Wigner plane? The following section is devoted to this question. However, instead of operators on $L^{2}(\mathbb{R})$, we consider operators acting on $L^{2}\left(\mathbb{R}^{n}\right)$, since finding a solution for the $n$-dimensional problem also yields a solution for the one-dimensional problem, but it does not follow straightforwardly from the solution of the one-dimensional case.

\section{AFFINE TRANSFORMATIONS IN THE WIGNER PLANE}

Inspired by the FRFT and its action in the Wigner plane, we search for linear operators $\mathscr{V}$ on $L^{2}\left(\mathbb{R}^{n}\right)$ such that there exist a matrix $A \in \mathbb{R}^{n \times n}$ and a vector $b \in \mathbb{R}^{n}$ for which

$$
\mathscr{W} \mathscr{V}[\mathscr{V} f](x, \omega)=\mathscr{W} \mathscr{V}[f](A(x, \omega)+b)
$$

holds for all $f \in L^{2}\left(\mathbb{R}^{n}\right)$. Since the translation vector $b$ is the result of the unitary operator $\mathcal{N}_{-b}$ (see (4)), it suffices to search for linear operators $\mathscr{V}$ on $L^{2}\left(\mathbb{R}^{n}\right)$ such that there exists a matrix $A \in \mathbb{R}^{2 n \times 2 n}$ for which

$$
\mathscr{W} \mathscr{V}[\mathscr{V} f](x, \omega)=\mathscr{W} \mathscr{V}[f](A(x, \omega)) .
$$

Furthermore, we restrict ourselves to matrices $A$ for which $\operatorname{det} A= \pm 1$. Operators that yield such transformations $A$ in phase space preserve energy which follows straightforwardly from (6) and (13) by substitution of variables.

In a previous paper [9], we dealt with the problem of classifying all unitary operators on $L^{2}\left(\mathbb{R}^{n}\right)$ that correspond to a matrix $A \in \mathbb{R}^{2 n \times 2 n}$ in the sense of (13). Moreover, by polarization, this class of unitary operators will also satisfy

$$
\mathscr{W} \mathscr{V}[\mathscr{V} f, \mathscr{V} g](x, \omega)=\mathscr{W} \mathscr{V}[f, g](A(x, \omega)),
$$

for all $f, g \in L^{2}\left(\mathbb{R}^{n}\right)$.

In [10], it has been shown that a necessary and sufficient condition on the matrix $A$, such that a unitary operator $\mathscr{V}$ exists, is that $A \in \mathbb{R}^{2 n \times 2 n}$ is symplectic. This means that given the $2 \times 2$ block decomposition

$$
A=\left(\begin{array}{ll}
A_{11} & A_{12} \\
A_{21} & A_{22}
\end{array}\right),
$$

the following relations should hold:

$$
\begin{gathered}
A_{22}^{T} A_{11}-A_{12}^{T} A_{21}=I_{n}, \\
A_{11}^{T} A_{21}=A_{21}^{T} A_{11}, \\
A_{22}^{T} A_{12}=A_{12}^{T} A_{22} .
\end{gathered}
$$


It can also be shown [11] that for symplectic matrices, we have $\operatorname{det} A=1$. In the sequel of this paper, we use the notation $\operatorname{Sp}(n)$ for all real-valued symplectic $2 n \times 2 n$ symplectic matrices.

Starting with a symplectic matrix $A \in \mathbb{R}^{2 n \times 2 n}$, we derived in [9] an integral representation for a unitary operator $\mathscr{F}_{A}$ on $L^{2}\left(\mathbb{R}^{n}\right)$ that satisfies (14). This operator is defined as follows.

Definition 1. Let $A \in \mathrm{Sp}(n)$ with block decomposition (15). Then for $A_{12}=0$, the linear operator $\mathscr{F}_{A}$ on $L^{2}\left(\mathbb{R}^{n}\right)$ is given by

$$
\mathscr{F}_{A}[f](x)=\sqrt{\left|\operatorname{det} A_{11}\right|} e^{-i\left(A_{11}^{T} A_{21} x, x\right) / 2} f\left(A_{11} x\right) .
$$

Furthermore, if $A_{12} \neq 0$, then

$$
\begin{aligned}
\mathscr{F}_{A}[f](x) & =C_{A} e^{-i\left(A_{11}^{T} A_{21} x, x\right) / 2} \\
& \times \int_{\operatorname{Ran}\left(A_{12}^{T}\right)} f\left(A_{12} t+A_{11} x\right) e^{-i\left(A_{12}^{T} A_{22} t, t\right) / 2-i\left(t, A_{12}^{T} A_{21} x\right)} d t,
\end{aligned}
$$

for all $f \in L^{2}\left(\mathbb{R}^{n}\right)$ and with

$$
C_{A}=\sqrt{\frac{s\left(A_{12}\right)}{(2 \pi)^{d} \operatorname{vol}_{\operatorname{Ker}\left(A_{12}\right)}\left(A_{22}\right)}} .
$$

Here $s\left(A_{12}\right)$ denotes the product of the nonzero singular values of $A_{12}$, and $\operatorname{vol}_{\operatorname{Ker}\left(A_{12}\right)}\left(A_{22}\right)$ denotes the volume of the simplex spanned by $A_{22} e_{1}, \ldots, A_{22} e_{n}$, with $e_{1}, \ldots, e_{n}$ any orthonormal basis in the null space of $A_{12}$.

In the particular case for which $A_{12}$ is nonsingular, we have $\operatorname{vol}_{\operatorname{Ker}\left(A_{12}\right)}\left(A_{22}\right)=1$ and $s\left(A_{12}\right)=\operatorname{det}\left(A_{12}\right)$. Furthermore, using the substitution $u=A_{12} t+A_{11} x$ and conditions (16), formula (18) is simplified to

$$
\begin{aligned}
\mathscr{F}_{A}[f](x)= & \frac{e^{-i\left(A_{22} A_{12}^{-1} x, x\right) / 2}}{(2 \pi)^{n / 2} \sqrt{\left|\operatorname{det} A_{12}\right|}} \\
& \times \int_{\mathbb{R}^{n}} f(u) e^{-i\left(\left(A_{12}^{-1} A_{11} u, u\right) / 2-\left(x, A_{12}^{-1} u\right)\right)} d u
\end{aligned}
$$

which corresponds to the metaplectic representation of $\mathrm{Sp}(n)$, as given in [11].

The multidimensional FRFT is a special case of (20), namely, it follows from (20) by taking

$$
\begin{aligned}
& A_{11}=A_{22}=\operatorname{diag}\left(\cos \alpha_{1}, \ldots, \cos \alpha_{n}\right), \\
& A_{12}=\operatorname{diag}\left(-\sin \alpha_{1}, \ldots,-\sin \alpha_{n}\right)
\end{aligned}
$$

if $\alpha_{i} \neq 2 k \pi$, for all $i=1, \ldots, n$. Moreover, the FRFT can also be seen as a special case of the operator

$$
\mathscr{F}_{\Gamma, \Delta}[f](x)=\frac{e^{i(\Gamma x, x) / 2}}{(2 \pi)^{n / 2} \sqrt{|\operatorname{det} \Delta|}} \int_{\mathbb{R}^{n}} f(u) e^{i\left((\Gamma u, u) / 2-\left(x, \Delta^{-1} u\right)\right)} d u,
$$

with $\Gamma \in \mathbb{R}^{n \times n}$ symmetric and $\Delta \in \mathbb{R}^{n \times n}$ with $\operatorname{det} \Delta \neq 0$. For simplicity, we also assume $\Delta$ to be symmetric. Of course this operator is also a special case of (18). A generalization of the FRFT in this way was already suggested in [12].

\section{LOCALIZATION PROBLEMS AND THE METAPLECTIC REPRESENTATION}

In this section, we consider the celebrated problem in signal processing of maximizing energy in both time and frequency, or space and frequency in more dimensions. This problem has already received much attention in the literature, see, for example, $[13,14,15,16]$.

We will show how the representation formula (18) can be used to solve a whole class of localization problems if only one problem of this class has already been solved. In the problems we consider here, the goal is to find a function $f \in L^{2}\left(\mathbb{R}^{n}\right)$ that maximizes

$$
\int_{\mathbb{R}^{n}} \int_{\mathbb{R}^{n}} \sigma(x, \omega)^{\mathscr{W}} \mathcal{V}[f](x, \omega) d x d \omega
$$

for some bounded weight function $\sigma$, called the symbol. Consequently, if

$$
\sigma(x, \omega)=\mathbf{1}_{\Omega}(x, \omega)= \begin{cases}1, & (x, \omega) \in \Omega, \\ 0, & \text { otherwise }\end{cases}
$$

with $\Omega \subset \mathbb{R}^{2 n}$, then (23) represents the energy of $f$ in the Wigner plane within the region $\Omega$.

For solving this maximum energy problem, we introduce the localization operator $\mathscr{L}(\sigma)$ by

$$
(\mathscr{L}(\sigma) f, g)=\int_{\mathbb{R}^{n}} \int_{\mathbb{R}^{n}} \sigma(x, \omega)^{\mathscr{W}} \mathcal{V}[f, g](x, \omega) d x d \omega,
$$

for all $f, g \in L^{2}\left(\mathbb{R}^{n}\right)$. Note that by introducing this operator $\mathscr{L}(\sigma)$, the problem comes down to search for such functions $f$ that maximize $(\mathscr{L}(\sigma) f, f)$. The association of a symbol $\sigma$ with the localization operator $\mathscr{L}(\sigma)$ is called the Weyl correspondence, see, for example, [11, 17]. In [14], Flandrin showed that $\mathscr{L}(\sigma)$ is self-adjoint for real-valued $\sigma$. Moreover, it was shown in [18] that if $\sigma$ is real valued and of finite energy, absolutely integrable, or just bounded, then the eigenvectors of $\mathscr{L}(\sigma)$ can be chosen to form an orthonormal basis for $L^{2}\left(\mathbb{R}^{n}\right)$, the set of real-valued eigenvalues is countable, and the possible accumulation point is 0 . The function $f_{\max }$ that maximizes (23) is given by the eigenvector $\phi_{0}$ of $\mathscr{L}(\sigma)$ corresponding to the largest eigenvalue $\lambda_{0}$ of $\mathscr{L}(\sigma)$.

We now assume that for a certain symbol $\sigma \in L^{\infty}\left(\mathbb{R}^{2 n}\right)$, the function that maximizes (23), $f_{\max }$, and its corresponding fraction of energy $\lambda_{0}$ are known. Then the following lemma gives us the solutions for a whole class of localization problems.

Lemma 1. Let $\sigma \in L^{\infty}\left(\mathbb{R}^{2 n}\right), \mathscr{L}(\sigma)$ the localization operator as defined in (25), and $A \in \mathrm{Sp}(n)$. Then $\mathscr{F}_{A} \phi_{k}, k \in \mathbb{N}$, and $\lambda_{k}, k \in \mathbb{N}$, are, respectively, the eigenvectors and eigenvalues of $\mathscr{L}(\sigma \circ A)$. Here $\phi_{k}, k \in \mathbb{N}$ and $\lambda_{k}, k \in \mathbb{N}$ denote, respectively, the eigenvectors and eigenvalues of $\mathscr{L}(\sigma)$. 
Proof. The proof follows straightforwardly from definition (25) and property (14). We have

$$
\begin{aligned}
\left(\mathscr{F}_{A}\right. & \left.\mathscr{L}(\sigma) \mathscr{F}_{A}^{*} f, g\right) \\
& =\left(\mathscr{L}(\sigma) \mathscr{F}_{A}^{*} f, \mathscr{F}_{A}^{*} g\right) \\
& =\int_{\mathbb{R}^{n}} \int_{\mathbb{R}^{n}} \sigma(x, \omega)^{\mathscr{W}} \mathscr{V}\left[\mathscr{F}_{A}^{*} f, \mathscr{F}_{A}^{*} g\right](x, \omega) d x d \omega \\
& =\int_{\mathbb{R}^{n}} \int_{\mathbb{R}^{n}} \sigma(x, \omega)^{\mathscr{W}} \mathscr{V}[f, g]\left(A^{-1}(x, \omega)\right) d x d \omega \\
& =\int_{\mathbb{R}^{n}} \int_{\mathbb{R}^{n}} \sigma(A(x, \omega))^{\mathscr{W}}[f, g](x, \omega) d x d \omega \\
& =(\mathscr{L}(\sigma \circ A) f, g) .
\end{aligned}
$$

Now, assume that $\left\{\phi_{k} \mid k \in \mathbb{N}\right\}$ is the set of eigenvectors of $\mathscr{L}(\sigma)$ and $\left\{\lambda_{k} \mid k \in \mathbb{N}\right\}$ the set of corresponding eigenvectors. Then

$$
\begin{aligned}
\mathscr{L}\left(\sigma_{A}\right) \mathscr{F}_{A} \phi_{k} & =\left(\mathscr{F}_{A} \mathscr{L}(\sigma) \mathscr{F}_{A}^{*}\right) \mathscr{F}_{A} \phi_{k} \\
& =\mathscr{F}_{A} \mathscr{L}(\sigma) \phi_{k}=\lambda_{k} \mathscr{F}_{A} \phi_{k},
\end{aligned}
$$

which completes the proof.

For one-dimensional problems, the following corollary applies.

Corollary 1. Let $\Omega \subset \mathbb{R}^{2}$ be an arbitrary bounded region in the Wigner plane and let $f_{\max } \in L^{2}(\mathbb{R})$ be the signal that has maximal energy $E_{\max }$ in $\Omega$. Then the signal that has maximal energy $E_{\max }$ in $\Omega^{\prime}=A(\Omega)-b$ is given by $\mathcal{N}_{b} \mathscr{F}_{A} f_{\max }$ with $\mathcal{N}_{b}$, $b \in \mathbb{R}$ as in (4) and $A \in \mathbb{R}^{2 \times 2}$ with $\operatorname{det} A=1$.

To illustrate Corollary 1 , the previous result is now applied to two well-known energy localization problems.

\section{EXAMPLES}

The two examples we discuss in this section are the maximization of energy on ellipsoidal areas in the Wigner plane and on parallelograms in the time-frequency plane that is related to the Rihaczek distribution. Both problems have already been studied in the literature $[14,19]$ using traditional results on the Wigner distribution. Here we present a way of solving these problems using a generalization of the FRFT. For simplicity, we restrict ourselves to the case of onedimensional signals, where the idea of using the fractional transform for solving such problems can also be visualized in a better way.

\subsection{Energy concentration on ellipsoidals in the Wigner plane}

The problem we consider first is the concentration of energy in a circular region in the Wigner plane. So we consider a region

$$
C_{R}=\left\{(x, \omega) \in \mathbb{R}^{2} \mid x^{2}+\omega^{2} \leq R\right\}
$$

and search for functions $f \in L^{2}(\mathbb{R})$, with normalized energy $\|f\|_{L^{2}}$, for which

$$
E_{f}(R)=\int_{C_{R}} \frac{\mathscr{W} \mathscr{V}[f](x, \omega) d x d \omega}{\|f\|^{2}}
$$

is maximized. For solving this localization problem, we observe that

$$
E_{f}(R)=\left(\mathscr{L}\left(\mathbf{1}_{C_{R}}\right) f, f\right)
$$

with $\mathscr{L}$ the localization operator $\mathscr{L}(\sigma)$ as in $(25)$.

We observe that $\mathbf{1}_{C_{R}}$ is a bounded real-valued symbol, and so we have an orthonormal basis of eigenfunctions with the operator $\mathscr{L}\left(\mathbf{1}_{C_{R}}\right)$ and corresponding positive eigenvalues. The function $f_{\max }$, that maximizes $E_{f}(R)$, is then given by the eigenvector $\phi_{0}$ of $\mathscr{L}\left(\mathbf{1}_{C_{R}}\right)$ corresponding to the largest eigenvalue $\lambda_{0}$ of $\mathscr{L}\left(\mathbf{1}_{C_{R}}\right)$. Moreover, $E_{\max }(R)$ is given by $\lambda_{0}$.

The eigenvectors of $\mathscr{L}\left(\mathbf{1}_{C_{R}}\right)$ are given by the Hermite functions $H_{k}, k \in \mathbb{N}$, which is a result by Janssen in [20]. Furthermore, it can be shown [19] that the corresponding eigenvalues satisfy

$$
\begin{aligned}
& \lambda_{0}=1-e^{-R^{2}}, \\
& \lambda_{k+1}=\lambda_{k}-(-1)^{k} e^{-R^{2}}\left(L_{k}\left(2 R^{2}\right)-L_{k+1}\left(2 R^{2}\right)\right),
\end{aligned}
$$

where $k \in \mathbb{N} \backslash\{0\}$ with $L_{k}$ being the Laguerre polynomial of degree $k$. It can be shown that $\lambda_{0} \geq \lambda_{k}, k \in \mathbb{N}$, see [20]. Consequently, $E_{\max }(R)=1-e^{-R^{2}}$ and $f_{\max }(x)=H_{0}(x)=$ $e^{-x^{2} / 2}$.

The circular region can also be translated over a vector $\left(x_{0}, \omega_{0}\right)$. As a result of $(4)$, the eigenfunctions of $\mathscr{L}(\sigma)$ are then given by $\mathcal{N}_{\left(x_{0}, \omega_{0}\right)} H_{k}$. The eigenvalues remain the same.

Dilating circular regions in either the time or frequency direction will yield ellipsoidal regions that are orientated along one of these axes. The total class of ellipsoidal regions that are obtained from a circle by means of an area preserving affine transformation is given by $A\left(C_{R}\right)-b$, with $A \in \mathbb{R}^{2 \times 2}$, $\operatorname{det} A= \pm 1$, and $b \in \mathbb{R}^{2 n}$. We restrict ourselves to the case $\operatorname{det} A=1$ since a function that maximizes energy in the regions $A\left(C_{R}\right)-b$, with $\operatorname{det} A=-1$, is the complex conjugate of the function that maximizes energy in the regions $M A\left(C_{R}\right)-b$, with

$$
M=\left(\begin{array}{cc}
1 & 0 \\
0 & -1
\end{array}\right)
$$

Furthermore, since symplectic matrices in $\mathbb{R}^{2 \times 2}$ are matrices with $\operatorname{det} A=1$, Corollary 1 applies to this situation, which means that the eigenfunctions of $\mathscr{L}\left(\mathbf{1}_{A\left(C_{R}\right)-b}\right)$ are given by $\mathcal{N}_{b} \mathscr{F}_{A} H_{k}$ and that its eigenvalues satisfy the recursive relations for the eignvalues as presented above. Particularly, we solved the following energy localization problem. Let $\tilde{C}_{R}$ be the ellipsoidal region given by

$$
\tilde{C}_{R}=A\left(C_{R}\right)-b,
$$

with $A \in \mathbb{R}^{2 \times 2}$ and $b \in \mathbb{R}$, then $\mathcal{N}_{b} \mathscr{F}_{A} H_{0}$ is the signal that has maximal energy $E_{\max }(R)=1-e^{-R^{2}}$ in this region of the Wigner plane. 


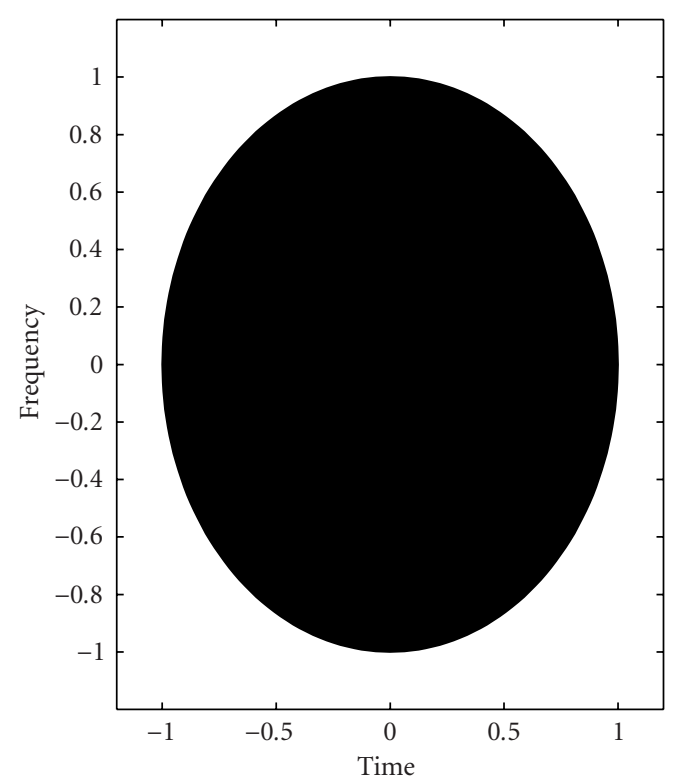

(a)

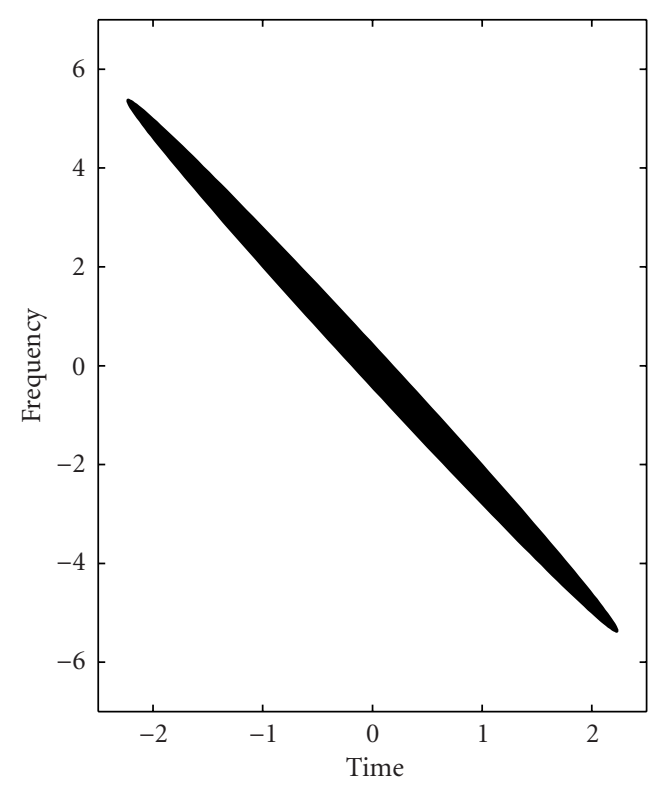

(c)

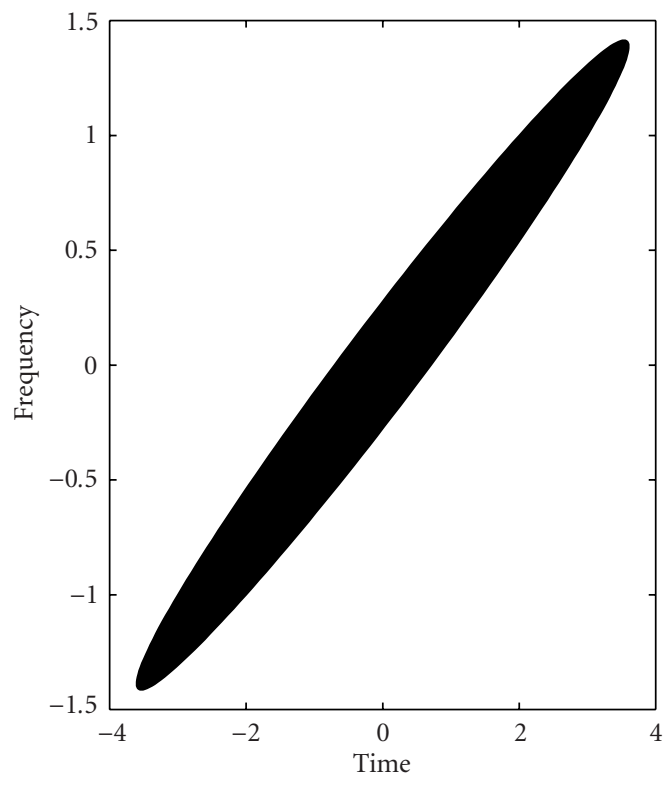

(b)

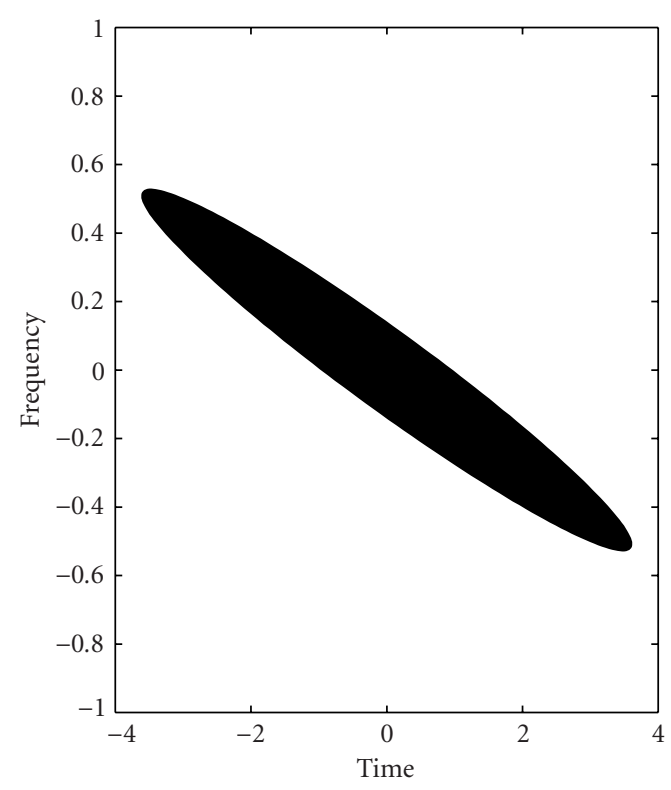

(d)

FIgURE 1: Localization on a circle/ellipse: (a) the circular region and (b), (c), (d) ellipsoidal regions $A(\Omega)$ for different $A \in \mathbb{R}^{2 \times 2}$, where $\operatorname{det} A=1$.

Figure 1 illustrates the type of regions one can obtain by starting with the circle $C_{1}$ and then transforming it by a symplectic matrix $A$. In this example, we have chosen

$$
\begin{gathered}
A=\left(\begin{array}{ll}
3 & 2 \\
1 & 1
\end{array}\right), \quad A=\left(\begin{array}{cc}
2 & 1 \\
-5 & -2
\end{array}\right), \\
A=\left(\begin{array}{cc}
-3 & 2 \\
\frac{1}{2} & -\frac{1}{6}
\end{array}\right),
\end{gathered}
$$

for the domains (b), (c), and (d), respectively. Note that the maximal amount of energy a signal can have in each of these regions is $(e-1) / e$.

\subsection{Energy concentration on parallelograms in the Rihaczek plane}

The second problem we consider is the maximization of a signal $f \in L^{2}(\mathbb{R})$, normalized to energy equal to 1 , within a rectangular plane in phase space, with respect to the Rihaczek 
distribution

$$
\mathscr{R}[f](x, \omega)=\frac{f(x) \overline{\hat{f}(\omega)} e^{-i \omega x}}{\sqrt{2 \pi}} .
$$

This problem can also be related to the problem of maximizing energy with the localization operator $\mathscr{L}(\sigma)$. To show this, we introduce the mixed Rihaczek distribution $\mathscr{R}[f, g]$ by

$$
\mathscr{R}[f, g](x, \omega)=\frac{f(x) \overline{\hat{g}(\omega)} e^{-i \omega x}}{\sqrt{2 \pi}} .
$$

We will show that

$$
(\mathscr{L}(\sigma) f, g)=\int_{-\omega_{0}}^{\omega_{0}} \int_{-x_{0}}^{x_{0}} \mathscr{R}[f, g](x, \omega) d x d \omega,
$$

for all signals $f$ and $g$ with finite energy if

$$
\sigma=\mathbf{1}_{\left[-x_{0}, x_{0}\right] \times\left[-\omega_{0}, \omega_{0}\right]} * \varphi
$$

for some $x_{0}, \omega_{0} \in \mathbb{R}^{+}$and where $\varphi$ is given by $\varphi(x, \omega)=$ $e^{-2 i x \omega}$. We observe that $\|\sigma\|_{\infty} \leq 1$, and so $\sigma$ is a bounded symbol.

To prove relation (36), we first write $(\mathscr{L}(\sigma) f, g)$ as the inner product

$$
\begin{aligned}
(\mathscr{L}(\sigma) f, g) & =\left(\sigma_{0} * \varphi, \mathscr{W} \mathscr{V}[f, g]\right) \\
& =\left(\sigma_{0}, \varphi * W^{\mathscr{V}}[f, g]\right)
\end{aligned}
$$

with $\sigma_{0}=\mathbf{1}_{\left[-x_{0}, x_{0}\right] \times\left[-\omega_{0}, \omega_{0}\right]}$. The latter expression can be rewritten as

$$
\begin{aligned}
(\varphi * \mathscr{W} \mathscr{V}[f, g])(x, \omega) & \\
= & \frac{1}{2 \pi^{2}} \int_{\mathbb{R}} \int_{\mathbb{R}} \int_{\mathbb{R}} \varphi(p, q) f(x-p+t) \overline{g(x-p-t)} \\
& \times e^{-2 i t(\omega-q)} d t d p d q \\
= & \frac{1}{2 \pi^{2}} \int_{\mathbb{R}} \int_{\mathbb{R}} \int_{\mathbb{R}} \varphi\left(-\frac{u+v}{2}, q\right) f(x+u) \overline{g(x+v)} \\
= & \frac{1}{4 \pi^{2}} \int_{\mathbb{R}} \int_{\mathbb{R}} \int_{\mathbb{R}} e^{-i q x} f(u) \overline{g(v)} e^{-i u(\omega-q)} e^{i v \omega} d u d v d q \\
= & \frac{1}{2 \pi} \int_{\mathbb{R}} e^{-i q x} \hat{f}(\omega-q) \overline{\hat{g}(\omega)} d q \\
= & \frac{1}{2 \pi} e^{-i \omega x} \overline{\hat{g}(\omega)} \int_{\mathbb{R}} e^{-i \omega x} \hat{f}(q) e^{i q x} d q \\
= & \frac{f(x) \bar{g}(\omega) e^{-i \omega x}}{\sqrt{2 \pi}},
\end{aligned}
$$

yielding relation (36).

We observe that the mixed Wigner distribution reduces to the Rihaczek distribution for $g=f$. This means that $\mathscr{L}(\sigma)$ is the localization operator that corresponds to the rectangular region $\left[-x_{0}, x_{0}\right] \times\left[-\omega_{0}, \omega_{0}\right]$ in the Rihaczek plane, that is, the time-frequency plane generated by the Rihaczek distribution.

As far as known, no explicit solution exists for the eigenvector/value problem for this $\mathscr{L}(\sigma)$. However, some information of this $\mathscr{L}(\sigma)$ can be obtained by looking at $\mathscr{L}(\sigma)^{*} \mathscr{L}(\sigma)$, with $\mathscr{L}(\sigma)^{*}$ the adjoint of $\mathscr{L}(\sigma)$. Observe that the eigenvalues of $\mathscr{L}(\sigma) * \mathscr{L}(\sigma)$ are directly related to the singular values of $o p L(\sigma)$. For studying $\mathscr{L}(\sigma)^{*} \mathscr{L}(\sigma)$, we consider a result by Flandrin. In [14], it was shown that when $\sigma$ is as in (37), then $\mathscr{L}(\sigma)=\mathscr{B}\left(\omega_{0}\right) \mathscr{P}\left(x_{0}\right)$, with

$$
\begin{aligned}
& \mathscr{B}\left(\omega_{0}\right)[f](x)=\sqrt{\frac{2}{\pi}} \int_{\mathbb{R}} \frac{\sin \left(\omega_{0}(x-u)\right)}{(x-u)} f(u) d u, \\
& \mathscr{P}\left(x_{0}\right)[f](x)= \begin{cases}f(x), & \text { if }|x| \leq x_{0}, \\
0, & \text { if }|x|>x_{0} .\end{cases}
\end{aligned}
$$

These projections have been studied extensively by Slepian and Pollak $[16,21]$. In particular, they showed that the eigenfunctions of the operator $\mathscr{P}\left(x_{0}\right) \mathscr{B}\left(\omega_{0}\right) \mathscr{P}\left(x_{0}\right)$ are given by the prolate spheroidal wave functions (PSWF) $\psi_{k}, k \in \mathbb{N}$, (see [22]) and their corresponding eigenvalues depend on the product $x_{0} \omega_{0}$. Moreover, for $x_{0} \omega_{0} \rightarrow \infty$ approximately, the first $2 x_{0} \omega_{0} / \pi$ eigenvalues that correspond to the PSWF attain a value close to unity. For index numbers in a region around $2 x_{0} \omega_{0} / \pi$, the eigenvalues plunge to zero and attain values close to zero afterwards. The number of eigenvalues in the region where the eigenvalues decrease from close to one to close to zero is proportional to $\log x_{0} \omega_{0}$. This asymptotical behaviour has been described rigorously in [21].

Furthermore, we observe that the singular values of $\mathscr{L}(\sigma)$ are given by $s_{k}=\sqrt{\lambda_{k}}$, where $\lambda_{k}$ denote the eigenvalues of the operator $\mathscr{P}\left(x_{0}\right) \mathscr{B}\left(\omega_{0}\right) \mathscr{P}\left(x_{0}\right)$. By definition, its asymptotical behavior is similar to the behaviour of the eigenvalues of $\mathscr{P}\left(x_{0}\right) \mathscr{B}\left(\omega_{0}\right) \mathscr{P}\left(x_{0}\right)$.

The eigenvectors of $\mathscr{L}(\sigma) * \mathscr{L}(\sigma)$ are given by the PSWF. However, they do not give rise to explicit expressions for the eigenfunctions of $\mathscr{L}(\sigma)$.

As for the circular regions in the Wigner plane, we can also apply a linear transformation $A \in \mathbb{R}^{2 \times 2}$, with $\operatorname{det} A=$ 1 , and a translation over $b \in \mathbb{R}^{2}$ on the rectangular region in the Rihaczek plane. This leads to parallelograms $A\left(\left[-x_{0}, x_{0}\right] \times\left[-\omega_{0}, \omega_{0}\right]\right)-b$. Figure 2 illustrates the type of regions one can obtain by starting with the rectangular $[-1,1] \times[-1,1]$ and then transforming it by the symplectic matrices $A$ as indicated in (33).

In a straightforward way, it can be shown that Lemma 1 also holds for the operator $\mathscr{L}(\sigma)^{*} \mathscr{L}(\sigma)$ and so also Corollary 1 holds for $\mathscr{L}(\sigma)^{*} \mathscr{L}(\sigma)$. For this situation, it means that the singular values of the operator $\mathscr{L}\left(\mathbf{1}_{A\left(\left[-x_{0}, x_{0}\right] \times\left[-\omega_{0}, \omega_{0}\right]\right)-b}\right)$ are given by $\sqrt{\lambda_{k}}$, where $\lambda_{k}$ satisfies the previous discussed asymptotical behaviour. The eigenfunctions of

$$
\mathscr{L}\left(\mathbf{1}_{A\left(\left[-x_{0}, x_{0}\right] \times\left[-\omega_{0}, \omega_{0}\right]\right)}\right)^{*} \mathscr{L}\left(\mathbf{1}_{A\left(\left[-x_{0}, x_{0}\right] \times\left[-\omega_{0}, \omega_{0}\right]\right)}\right)
$$

are given by $\mathcal{N}_{b} \mathscr{F}_{A} \psi_{k}$, with $\psi_{k}$ the PSWF. 


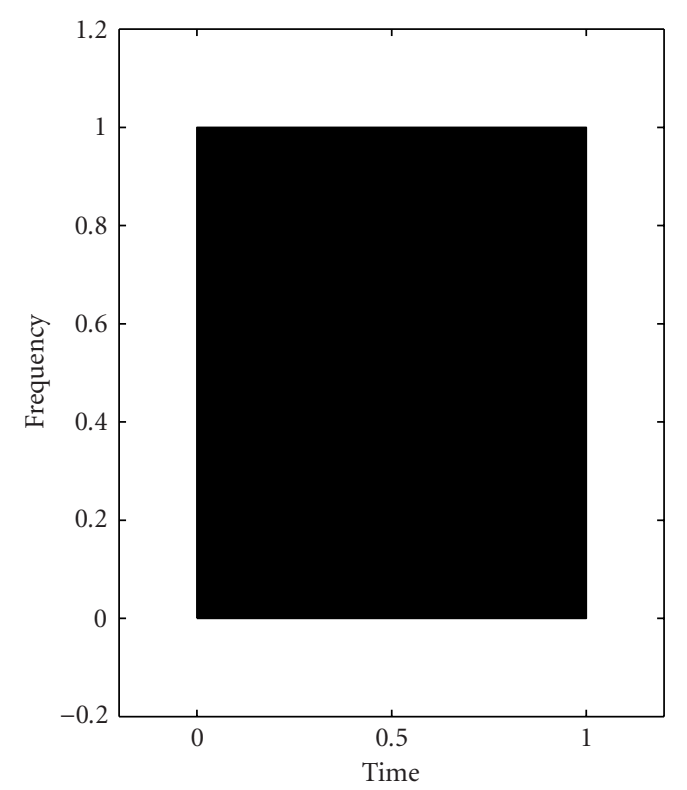

(a)

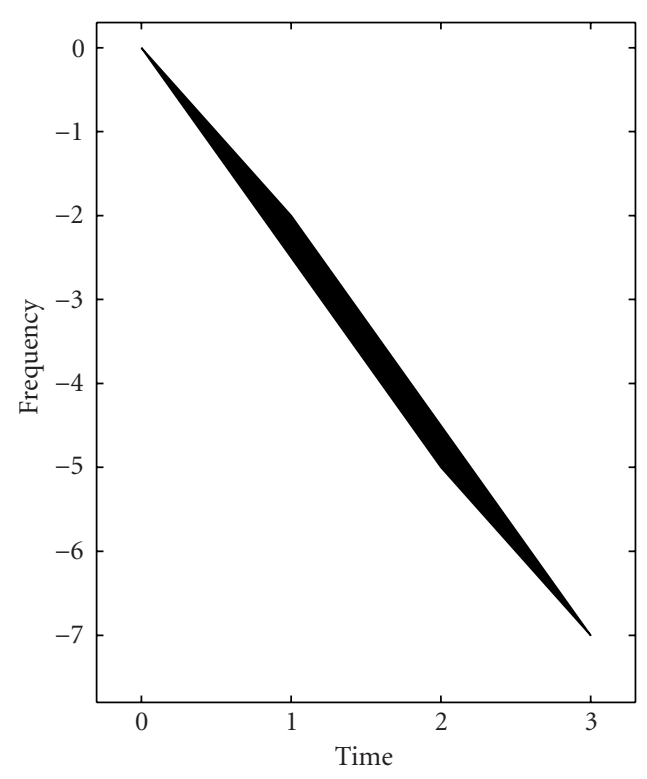

(c)

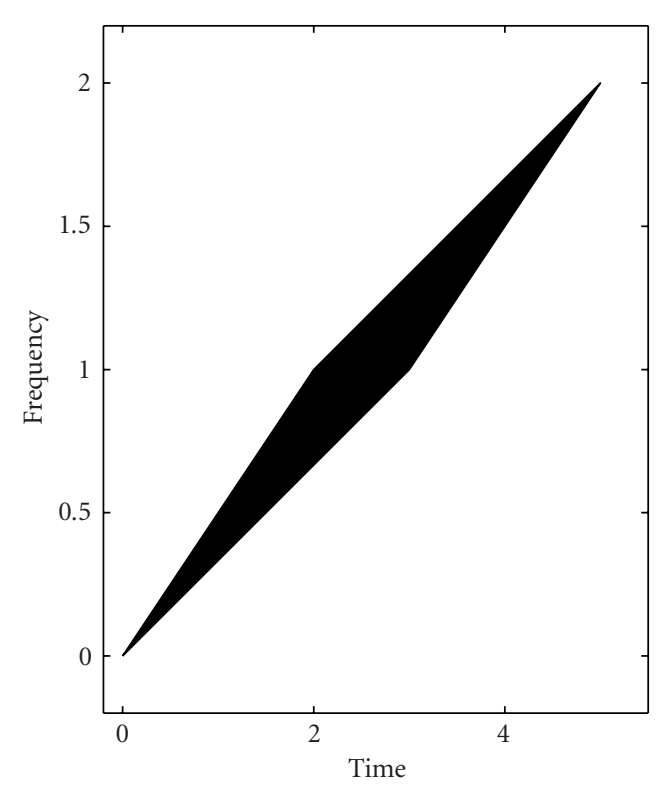

(b)

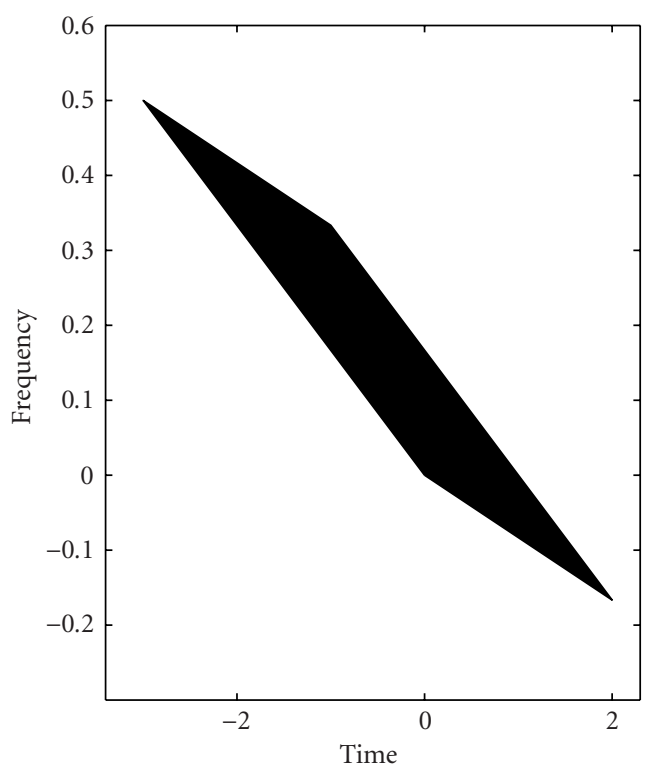

(d)

Figure 2: Localization on a rectangle/parallelogram: (a) the rectangular region $\Omega$ and (b), (c), (d) parallelograms $A(\Omega)$ for different $A \in$ $\mathbb{R}^{2 \times 2}$, where $\operatorname{det} A=1$.

\section{CONCLUSIONS}

In this paper, we have shown how a generalization of the $n$ dimensional FRFT can be used to analyze certain energy localization problems in the $2 n$-dimensional phase plane. This generalization is a newly derived representation of so-called metaplectic operators. These operators form a natural extension of the notion of the FRFT in the way that taking the Wigner distribution and a metaplectic operator in a cascade fashion corresponds to a symplectic transformation on the spatial and frequency parameters of the Wigner distribution.

The approach of solving localization problems with metaplectic operators (and their representation formula) has been illustrated by two classical examples in the onedimensional case. 
The presented integral representation formula is valid for all choices of the corresponding symplectic transformations in the Wigner plane. On the contrary, classical representation formulas [11] are only available for symplectic transformations with $2 \times 2$ block decompositions where not all four blocks are singular, which is the case if the metaplectic operator is a $d$-dimensional Fourier transform on $L^{2}\left(\mathbb{R}^{n}\right)$, with $0<d<n$.

\section{REFERENCES}

[1] H. Kober, "Wurzeln aus der Hankel-, Fourier- und aus anderen stetigen transformationen," Quart. J. Math. Oxford Ser., vol. 10, pp. 45-49, 1939.

[2] L. B. Almeida, "The fractional Fourier transform and timefrequency representations," IEEE Trans. Signal Processing, vol. 42, no. 11, pp. 3084-3091, 1994.

[3] S. C. Pei and J. J. Ding, "Relations between fractional operations and time-frequency distributions, and their applications," IEEE Trans. Signal Processing, vol. 49, no. 8, pp. 1638 $1655,2001$.

[4] T. A. C. M. Claasen and W. F. G. Mecklenbräuker, "The Wigner distribution-A tool for time-frequency signal analysis," Philips J. Res., vol. 35, no. 3, pp. 217-250, 1980.

[5] F. Hlawatsch and G. F. Boudreaux-Bartels, "Linear and quadratic time-frequency signal representations," IEEE Signal Processing Magazine, vol. 9, no. 2, pp. 21-67, 1992.

[6] V. Namias, "The fractional order Fourier transform and its application to quantum mechanics," J. Inst. Math. Appl., vol. 25, no. 3, pp. 241-265, 1980.

[7] F. H. Kerr, "Namias' fractional Fourier transforms on $L^{2}$ and applications to differential equations," J. Math. Anal. Appl., vol. 136, no. 2, pp. 404-418, 1988.

[8] A. C. McBride and F. H. Kerr, "On Namias's fractional Fourier transforms," IMA J. Appl. Math., vol. 39, no. 2, pp. 159-175, 1987.

[9] H. G. ter Morsche and P. J. Oonincx, "On the integral representations for metaplectic operators," J. Fourier Anal. Appl., vol. 8, no. 3, pp. 245-257, 2002.

[10] N. R. Wallach, Symplectic Geometry and Fourier Analysis, Math Sci Press, Brookline, Mass, USA, 1977.

[11] G. B. Folland, Harmonic Analysis in Phase Space, Princeton University Press, Princeton, NJ, USA, 1989.

[12] D. Mustard, "The fractional Fourier transform and the Wigner distribution," J. Austral. Math. Soc. Ser. B, vol. 38, no. 2, pp. 209-219, 1996.

[13] I. Daubechies, "Time-frequency localization operators: a geometric phase space approach," IEEE Transactions on Information Theory, vol. 34, no. 4, pp. 605-612, 1988.

[14] P. Flandrin, "Maximum signal energy concentration in a time-frequency domain," in Proc. IEEE Int. Conf. Acoustics, Speech, Signal Processing (ICASSP '88), vol. 4, pp. 2176-2179, NY, USA, April 1988.

[15] C. Heil, J. Ramanathan, and P. Topiwala, "Asymptotic singular value decay of time-frequency localization operators," in Wavelet Applications in Signal and Image Processing II, vol. 2303 of SPIE Proceedings, pp. 15-24, San Diego, Calif, USA, 1994.

[16] D. Slepian and H. O. Pollak, "Prolate spheroidal wave functions, Fourier analysis and uncertainty. I," Bell System Tech. J., vol. 40, pp. 43-63, 1961.

[17] J. C. T. Pool, "Mathematical aspects of the Weyl correspondence," J. Mathematical Phys., vol. 7, pp. 66-76, 1966.

[18] M. W. Wong, Weyl Transforms, Springer, NY, USA, 1998.
[19] P. Flandrin, Time-Frequency/Time-Scale Analysis, Academic Press, San Diego, Calif, USA, 1999.

[20] A. J. E. M. Janssen, "Positivity of weighted Wigner distributions,” SIAM J. Math. Anal., vol. 12, no. 5, pp. 752-758, 1981.

[21] D. Slepian, "Some comments on Fourier analysis, uncertainty and modeling," SIAM Rev., vol. 25, no. 3, pp. 379-393, 1983.

[22] P. M. Morse and H. Feschbach, Methods of Theoretical Physics, McGraw-Hill, London, UK, 1953.

Patrick J. Oonincx received his M.S. degree (with honors) in mathematics from Eindhoven University in 1995 with a thesis on generalizations of multiresolution analysis. In 2000, he received the Ph.D. degree in mathematics from University of Amsterdam. His thesis on the mathematics of joint time-frequency/scale analysis has also appeared as a textbook. From 2000 to 2002, he worked as a Postdoctoral Researcher on multiresolution image processing at the Research Institute for Mathematics and Computer Science (CWI) in Amsterdam. Currently, he works as an Assistant Professor in mathematics and signal processing at the Royal Netherlands Naval College, Den Helder, the Netherlands. His research interests are wavelet analysis, timefrequency signal representations, multiresolution imaging, and signal processing for underwater acoustics.

Hennie G. ter Morsche received his M.S. degree in 1967 from the University of Nijmegen in the field of nonlinear differential equations. From 1968 to 1978, he worked as a university teacher at the Technische Universiteit Eindhoven. Subsequently, he started at this university his Ph.D. research on spline functions. The thesis, entitled "Interpolational and extremal properties of Lspline functions," was completed in 1982.

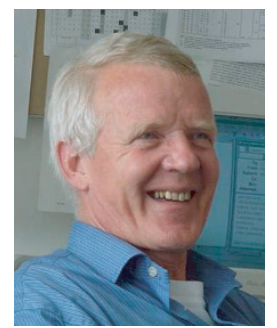
Half way through the eighties, his research interest has changed from splines to signal processing and wavelets. On this subject, he has written application oriented papers and a textbook, and guided several industrial projects. Nowadays, Dr. ter Morsche is the director of education of the bachelor and master course on applied and industrial mathematics in Eindhoven. 